$\underline{\text { Bolyai Society - Springer-Verlag }}$

\title{
AN ALGORITHM FOR PACKING NON-ZERO $A$-PATHS IN GROUP-LABELLED GRAPHS
}

\author{
MARIA CHUDNOVSKY, WILLIAM H. CUNNINGHAM, JIM GEELEN
}

Received September 9, 2004

Let $G=(V, E)$ be an oriented graph whose edges are labelled by the elements of a group $\Gamma$ and let $A \subseteq V$. An $A$-path is a path whose ends are both in $A$. The weight of a path $P$ in $G$ is the sum of the group values on forward oriented arcs minus the sum of the backward oriented $\operatorname{arcs}$ in $P$. (If $\Gamma$ is not abelian, we sum the labels in their order along the path.) We give an efficient algorithm for finding a maximum collection of vertex-disjoint $A$-paths each of non-zero weight. When $A=V$ this problem is equivalent to the maximum matching problem.

\section{Introduction}

Let $\Gamma$ be a group; we will use additive notation for groups, although they need not be abelian. A $\Gamma$-labelled graph is a graph $G$ in which each edge $e=u v \in E(G)$ is assigned weights $\omega_{G}(e, u), \omega_{G}(e, v) \in \Gamma$ where $\omega_{G}(e, u)=-\omega_{G}(e, v)$. Let $G$ be a $\Gamma$-labelled graph and let $A \subseteq V(G)$. An $A$-path is a path, with at least one edge, whose ends are both in $A$. Now, if $P=\left(v_{0}, e_{1}, v_{1}, e_{2}, v_{2}, \ldots, e_{k}, v_{k}\right)$ is a path in $G$, then the weight of $P$, denoted $\omega_{G}(P)$, is defined to be $\sum_{i=1}^{k} \omega_{G}\left(e_{i}, v_{i}\right)$.

We are interested in the maximum number of vertex-disjoint $A$-paths each of non-zero weight, which we denote by $\nu(G, A)$. Chudnovsky et al. [1] gave a min-max theorem for $\nu(G, A)$; they also discuss motivation for the

Mathematics Subject Classification (2000): 05C22

This research was partially conducted during the period Chudnovsky served as a Clay Mathematics Institute Long-Term Prize Fellow. The research was supported in part by the Natural Sciences and Engineering Council of Canada. 
non-zero $A$-paths problem. In particular, they show that Mader's $\mathcal{S}$-path problem [4] is a special case. The only previously known algorithm for Mader's $\mathcal{S}$-path problem was obtained by Lovász via a reduction to linear matroid matching [2]. We present an algorithm for finding a maximum collection of vertex-disjoint non-zero $A$-paths that runs in time $O\left(|V(G)|^{6}\right)$. In our complexity calculations, group operations (such as addition and comparison) are treated as elementary operations. Our algorithm is similar to an algorithm of Lovász and Plummer [3, p. 376] for finding a maximum matching. Lovász and Plummer cleverly abstract an algorithm from what would otherwise appear to be a nonconstructive proof of the EdmondsGallai Structure Theorem (see [3]). Using a similar approach, we obtain an algorithm from our proof of Theorem 1.3, which is a structure theorem for non-zero $A$-paths. Theorem 1.3 is closely related to a structure theorem of Sebő and Szegö [5] for Mader's $\mathcal{S}$-path problem; our results were, however, obtained independently.

Let $E_{0}(G, A)$ denote the set of all edges $e=u v \in E$ whose ends are both in $A$ and that have $\omega_{G}(e, v)=0$; note that deleting such edges does not affect $\nu$. Let $\operatorname{def}(G, A)=|A|-2 \nu(G, A)$; we call this the deficiency. Let odd $(G, A)$ denote the number of components $H$ of $G-E_{0}(G, A)$ with $|V(H) \cap A|$ odd. Finally let $X, A^{\prime} \subseteq V(G)$ such that $A \cup X \subseteq A^{\prime}$. It is straightforward to see that

$$
\begin{aligned}
\operatorname{def}(G, A) & \geq \operatorname{def}\left(G, A^{\prime}\right)-\left|A^{\prime}-A\right| \\
& \geq \operatorname{def}\left(G-X, A^{\prime}-X\right)-\left|A^{\prime}-A\right|-|X| \\
& \geq \operatorname{odd}\left(G-X, A^{\prime}-X\right)-\left|A^{\prime}-A\right|-|X| .
\end{aligned}
$$

Let $x \in V$ and let $\delta \in \Gamma$. We will construct a new $\Gamma$-labelled graph $G^{\prime}$ from $G$ by changing the labels as follows. For each edge $e=u v$ in $G$ we define

$$
\omega_{G^{\prime}}(e, u)= \begin{cases}\omega_{G}(e, u)+\delta, & \text { if } u=x \\ -\delta+\omega_{G}(e, u), & \text { if } v=x \\ \omega_{G}(e, u), & \text { otherwise. }\end{cases}
$$

We say that $G^{\prime}$ is obtained from $G$ by shifting by $\delta$ at $x$. Note that, if $x \notin A$, then this shift does not change the weight of any $A$-path (even when $\Gamma$ is non-abelian). If $G^{\prime}$ is a $\Gamma$-labelled graph obtained by a sequence of shifting operations on vertices not in $A$, then we say that $G$ and $G^{\prime}$ are $A$-equivalent. The main theorem in [1] is:

Theorem 1.1. Let $\Gamma$ be a group, let $G$ be a $\Gamma$-labelled graph, and let $A \subseteq V(G)$. Then there exists a $\Gamma$-labelled graph $G^{\prime}$ that is $A$-equivalent to $G$ and there exist sets $X, A^{\prime} \subseteq V(G)$ with $A \cup X \subseteq A^{\prime}$ such that

$$
\operatorname{def}(G, A)=\operatorname{odd}\left(G^{\prime}-X, A^{\prime}-X\right)-\left|A^{\prime}-A\right|-|X| .
$$


Our structure theorem provides a canonical choice for $A^{\prime}$ and $X$ in Theorem 1.1. Before stating the structure theorem we need some definitions; we start by clarifying our notation.

A path is a sequence $P=\left(v_{0}, e_{1}, v_{1}, e_{2}, v_{2}, \ldots, e_{k}, v_{k}\right)$ where $v_{0}, \ldots, v_{k}$ are distinct vertices of $G$ and $e_{i}$ has ends $v_{i-1}$ and $v_{i}$ for each $i \in\{1, \ldots, k\}$. Thus $P$ is ordered in that it has distinguished start $\left(v_{0}\right)$ and end $\left(v_{k}\right)$. The path $\left(v_{k}, e_{k}, v_{k-1}, \ldots, v_{1}, e_{1}, v_{0}\right)$ is denoted by $\bar{P}$. We allow paths consisting of a single vertex; we refer to such paths as trivial. We denote by $E(P)$ and $V(P)$ the set of edges and vertices of $P$, respectively.

An $A$-collection is a set $\Pi$ of vertex disjoint paths such that:

1. each vertex in $A$ is either the start or the end of a path in $\Pi$,

2. the start of each path $P \in \Pi$ is in $A$, and

3. if $P \in \Pi$ is non-trivial and has its end in $A$, then $\omega_{G}(P) \neq 0$.

A path $P \in \Pi$ is loose if it is trivial or its end is not in $A$; thus each path in $\Pi$ is either an $A$-path or it is loose (not both). The value of an $A$-collection $\Pi$, denoted $\operatorname{val}_{A}(\Pi)$ or $\operatorname{val}(\Pi)$, is the number of $A$-paths that it contains. The $A$-collection is optimal if $\operatorname{val}(\Pi)=\nu(G, A)$; note that there are optimal $A$ collections. Let $\mathcal{P}(G, A)$ denote the set of all $A$-collections and let $\mathcal{P}^{*}(G, A)$ denote the set of all optimal $A$-collections.

Given an $A$-collection $\Pi$, let $B_{A}(\Pi)$ (or $B(\Pi)$ ) denote the set of pairs $\left(v, \omega_{G}(P)\right)$ where $v$ is the end of a loose path $P \in \Pi$. Note that $|B(\Pi)|=$ $|A|-2 \operatorname{val}_{A}(\Pi)$. Now let $\mathcal{R}(G, A)=\cup\left(B(\Pi): \Pi \in \mathcal{P}^{*}(G, A)\right)$; the pairs in $\mathcal{R}(G, A)$ are called reachable pairs.

For each vertex $v \in V(G)$, we let $\Gamma(G, A, v)=\{\alpha \in \Gamma:(v, \alpha) \in \mathcal{R}(G, A)\}$. Now we let

$$
\begin{aligned}
D_{1}(G, A) & =\{v \in V(G):|\Gamma(G, A, v)|=1\}, \\
D_{2}(G, A) & =\{v \in V(G):|\Gamma(G, A, v)| \geq 2\}, \text { and } \\
D(G, A) & =D_{1}(G, A) \cup D_{2}(G, A) ;
\end{aligned}
$$

$D(G, A)$ is the set of reachable vertices. Note that $D_{1}(G, A)$ and $D_{2}(G, A)$ are not affected by shifting on a vertex $v \notin A$.

For $X \subseteq V(G)$, we let $N_{G}(X)$ denote the set of vertices in $V(G)-X$ that are adjacent to a vertex in $X$. To make use of the coming structure theorem, we need the following easy lemma.

Lemma 1.2. Let $G$ be a $\Gamma$-labelled graph and let $A \subseteq V(G)$. Then there exists a $\Gamma$-labelled graph $G^{\prime}$ that is $A$-equivalent to $G$ and such that:

(1) for each $v \in D_{1}\left(G^{\prime}, A\right), \Gamma\left(G^{\prime}, A, v\right)=\{0\}$, and 
(2) for each $u \in N_{G^{\prime}}\left(D\left(G^{\prime}, A\right)\right)-A$, there exists $u v=e \in E\left(G^{\prime}\right)$ such that $\omega_{G^{\prime}}(e, v) \in \Gamma\left(G^{\prime}, A, v\right)$.

Proof. Suppose that $v \in D_{1}(G, A)$ and that $\Gamma(G, A, v)=\{\alpha\}$. If $v \in A$, then $\alpha=0$. On the other hand, if $v \notin A$ and $G^{\prime}$ is obtained from $G$ by shifting by $-\alpha$ at $v$, then $\Gamma\left(G^{\prime}, A, v\right)=\{0\}$ and $\Gamma\left(G^{\prime}, A, y\right)=\Gamma(G, A, y)$ for all $y \in V(G)-\{v\}$.

Now suppose that $u v=e \in E(G)$ where $u \notin A \cup D(G, A)$ and $v \in D(G, A)$. Let $\alpha \in \Gamma(G, A, v)$ and let $G^{\prime}$ be the $\Gamma$-labelled graph obtained from $G$ by shifting by $\omega_{G}(e, v)-\alpha$ at $u$. Then $\omega_{G^{\prime}}(e, v)=\alpha$ and $\Gamma\left(G^{\prime}, A, y\right)=\Gamma(G, A, y)$ for all $y \in V(G)$.

We can now state our structure theorem.

Theorem 1.3. Let $\Gamma$ be a group, let $G$ be a $\Gamma$-labelled graph, and let $A \subseteq V(G)$. Now let $A^{\prime}=A \cup N_{G}(D(G, A)) \cup D_{1}(G, A)$ and let $X=$ $N_{G-E_{0}\left(G, A^{\prime}\right)}(D(G, A))$. If $(G, A)$ satisfies:

(1) for each $v \in D_{1}(G, A), \Gamma(G, A, v)=\{0\}$, and

(2) for each $u \in N_{G}(D(G, A))-A$, there exists $u v=e \in E(G)$ such that $\omega_{G}(e, v) \in \Gamma(G, A, v)$,

then $\operatorname{def}(G, A)=\operatorname{odd}\left(G-X, A^{\prime}-X\right)-\left|A^{\prime}-A\right|-|X|$.

\section{Proof of the structure theorem}

In this section we outline a proof of the structure theorem; this outline is intended to motivate the main steps in the algorithm. Throughout the rest of the paper we let $\Gamma$ be a group, we let $G$ be a group labelled graph, and we let $A \subseteq V(G)$.

It is an easy but important observation that the sets $D_{1}(G, A), D_{2}(G, A)$, and $\Gamma(G, A, v)$ are determined by $\mathcal{R}(G, A)$. This allows us to prove Theorem 1.3 inductively by changing $G$ and $A$ in ways that do not effect $\mathcal{R}(G, A)$. We begin with two easy observations:

2.1. If $u \in A-D(G, A)$, then $\nu(G-u, A-\{u\})=\nu(G, A)-1$ and $\mathcal{R}(G, A) \subseteq$ $\mathcal{R}(G-u, A-\{u\})$.

2.2. If $u \in V(G)-A$ and $\Gamma(G, A, u) \subseteq\{0\}$, then $\nu(G, A \cup\{u\})=\nu(G, A)$ and $\mathcal{R}(G, A) \subseteq \mathcal{R}(G, A \cup\{u\})$.

In the next two results we provide additional hypotheses to 2.1 and 2.2 so that the above inclusions hold with equality. We will not prove these lemmas now since they follow immediately from more general results (Lemmas 4.3 and 4.4) proved later. 
Lemma 2.3. Let $u \in A-D(G, A)$. If there exists $u v=e \in E(G)$ and $\alpha \in$ $\Gamma(G, A, v)$ such that $\omega_{G}(e, u) \neq-\alpha$, then $\nu(G-u, A-\{u\})=\nu(G, A)-1$ and $\mathcal{R}(G, A)=\mathcal{R}(G-u, A-\{u\})$.

Lemma 2.4. Let $u \in V(G)-A$ where $\Gamma(G, A, u) \subseteq\{0\}$. If there exists $u v=$ $e \in E(G)$ and $\alpha \in \Gamma(G, A, v)$ such that $\omega_{G}(e, u)=-\alpha$, then $\nu(G, A \cup\{u\})=$ $\nu(G, A)$ and $\mathcal{R}(G, A)=\mathcal{R}(G, A \cup\{u\})$.

With the main ingredients in place, we can begin the proof of the structure theorem. Suppose that:

(1) for each $v \in D_{1}(G, A), \Gamma(G, A, v)=\{0\}$, and

(2) for each $u \in N_{G}(D(G, A))-A$, there exists $u v=e \in E(G)$ such that $\omega_{G}(e, v) \in \Gamma(G, A, v)$.

Now let $A^{\prime}=A \cup N_{G}(D(G, A)) \cup D_{1}(G, A)$ and $X=N_{G-E_{0}\left(G, A^{\prime}\right)}(D(G, A))$.

Lemma 2.5. $\nu(G, A)=\nu\left(G-X, A^{\prime}-X\right)+|X|$ and $\mathcal{R}(G, A)=\mathcal{R}\left(G-X, A^{\prime}-X\right)$. Hence $\operatorname{def}(G, A)=\operatorname{def}\left(G-X, A^{\prime}-X\right)-\left|A^{\prime}-A\right|-|X|$.

Proof. Let $A^{\prime \prime}=A \cup N_{G}(D(G, A))$. First we consider $u \in A^{\prime \prime}-A$. By (2), there exists $u v=e \in E(G)$ such that $\omega_{G}(e, v) \in \Gamma(G, A, v)$. Then, by Lemma 2.4, $\nu(G, A \cup\{u\})=\nu(G, A)$ and $\mathcal{R}(G, A)=\mathcal{R}(G, A \cup\{u\})$. Hence $D(G, A)=$ $D(G, A \cup\{u\})$ and $A^{\prime \prime}=A \cup N_{G}(D(G, A))=A \cup N_{G}(D(G, A \cup\{u\}))$. Inductively we conclude that $\nu\left(G, A^{\prime \prime}\right)=\nu(G, A)$ and $\mathcal{R}\left(G, A^{\prime \prime}\right)=\mathcal{R}(G, A)$.

Now consider $u \in D_{1}(G, A)-A=A^{\prime}-A^{\prime \prime}$. By (1), we have $\Gamma(G, A, u)=\{0\}$ and, hence, $\Gamma\left(G, A^{\prime \prime}, u\right)=\{0\}$. Thus there exists $\Pi \in \mathcal{P}^{*}\left(G, A^{\prime \prime}\right)$ such that $(u, 0) \in B(\Pi)$. Let $P \in \Pi$ be the path ending at $u$, let $v$ be the vertex preceding $u$ on $P$, let $P_{v}$ be the initial subpath of $P$ ending at $v$, and let $e=u v$. Note that $\omega_{G}\left(P_{v}\right)-\omega_{G}(e, v)=\omega_{G}(P)=0$. Thus $\omega_{G}\left(P_{v}\right)=\omega_{G}(e, v)$. Let $\Pi_{v}=(\Pi-\{P\}) \cup\left\{P_{v}\right\}$. Now $\Pi_{v}$ is an optimal $A^{\prime \prime}$-collection with $\left(v, \omega_{G}\left(P_{v}\right)\right) \in B\left(\Pi_{v}\right)$. Therefore $\omega_{G}(e, v) \in \Gamma(G, A, v)$. Hence, by Lemma 2.4, $\nu\left(G, A^{\prime \prime} \cup\{u\}\right)=\nu\left(G, A^{\prime \prime}\right)=\nu(G, A)$ and $\mathcal{R}\left(G, A^{\prime \prime} \cup\{u\}\right)=\mathcal{R}\left(G, A^{\prime \prime}\right)=\mathcal{R}(G, A)$. Inductively this proves that $\nu(G, A)=\nu\left(G, A^{\prime}\right)$ and $\mathcal{R}(G, A)=\mathcal{R}\left(G, A^{\prime}\right)$.

Note that $X \subseteq A^{\prime}$. Now consider $u \in X$. By the definition of $X$, there exists $u v=e \in E(G)-E_{0}\left(G, A^{\prime}\right)$ where $v \in D\left(G, A^{\prime}\right)$. We claim that there exists $\alpha \in \Gamma\left(G, A^{\prime}, v\right)$ such that $\omega_{G}(e, v) \neq \alpha$; for this it suffices to consider $v \in D_{1}(G, A)$. In this case $\Gamma\left(G, A^{\prime}, v\right)=\{0\}$ and, since $u, v \in A^{\prime}, \omega_{G}(e, v) \neq 0$, as required. Therefore, by Lemma 2.3, $\nu\left(G-u, A^{\prime}-\{u\}\right)=\nu\left(G, A^{\prime}\right)-1$ and $\mathcal{R}\left(G, A^{\prime}\right)=\mathcal{R}\left(G-u, A^{\prime}-\{u\}\right)$. Inductively it follows that $\nu(G, A)=$ $\nu\left(G-X, A^{\prime}-X\right)+|X|$ and $\mathcal{R}(G, A)=\mathcal{R}\left(G-X, A^{\prime}-X\right)$, as required.

We need one more definition. A critical pair $(G, A)$ consists of a $\Gamma$ labelled graph $G$ and a set $A \subseteq V(G)$ such that $G$ is connected, $D_{1}(G, A)=A$, $D_{2}(G, A)=V(G)-A$, and $E_{0}(G, A)=\emptyset$. 
Now let $A_{1}=A^{\prime}-X$ and $G_{1}=G-X$. The next lemma follows from the definition of $G_{1}$ and $A_{1}$.

Lemma 2.6. For each component $H$ of $G_{1}-E_{0}\left(G_{1}, A_{1}\right)$, either $\operatorname{def}(H$, $\left.V(H) \cap A_{1}\right)=0$ or $\left(H, V(H) \cap A_{1}\right)$ is critical.

Proof. Let $G_{2}=G_{1}-E_{0}\left(G_{1}, A_{1}\right)$. By Lemma $2.5, \mathcal{R}\left(G_{1}, A_{1}\right)=\mathcal{R}(G, A)$ and, hence, $\mathcal{R}\left(G_{2}, A_{1}\right)=\mathcal{R}(G, A)$. Moreover $\mathcal{R}\left(G_{2}, A_{1}\right)$ is the union of the sets $\mathcal{R}\left(H, A_{1} \cap V(H)\right)$ taken over all components $H$ of $G_{2}$. Note that, if $u v=e \in E(G)$ with $u \in V(G)-D(G, A)$ and $v \in D(G, A)$, then either $u \in X$ or $e \in E_{0}\left(G, A^{\prime}\right)$. Thus, if $H$ is a component of $G_{2}$, then either $V(H) \subseteq D\left(G_{2}, A_{1}\right)$ or $V(H) \cap D\left(G_{2}, A_{1}\right)=\emptyset$. If $V(H) \cap D\left(G_{2}, A_{1}\right)=\emptyset$, then $\operatorname{def}\left(H, V(H) \cap A_{1}\right)=0$. Thus we may assume that $V(H) \subseteq D\left(G_{2}, A_{1}\right)$. Since $H$ is a component of $G_{2}$, $D_{1}\left(H, V(H) \cap A_{1}\right)=D_{1}\left(G_{2}, A_{1}\right) \cap V(H)$ and $D_{2}\left(H, V(H) \cap A_{1}\right)=D_{2}\left(G_{2}, A_{1}\right) \cap$ $V(H)$. By the definition of $A^{\prime}$, a vertex $v \in D\left(G_{2}, A_{1}\right)$ is in $D_{1}\left(G_{2}, A_{1}\right)$ if and only if $v \in A_{1}$. Hence $\left(H, V(H) \cap A_{1}\right)$ is critical, as required.

The final lemma was proved in [1]; we prove a more general lemma later (see 4.5).

Lemma 2.7. If $(G, A)$ is a critical pair, then $\operatorname{def}(G, A)=1$ and, hence, $|A|$ is odd.

It follows from Lemmas 2.6 and 2.7 that $\operatorname{def}\left(G_{1}, A_{1}\right)=\operatorname{odd}\left(G_{1}, A_{1}\right)$. Therefore

$$
\operatorname{def}(G, A)=\operatorname{odd}\left(G-X, A^{\prime}-X\right)-\left|A^{\prime}-A\right|-|X| .
$$

This completes the proof of the structure theorem.

\section{The exchange property}

Chudnovsky et al. [1] proved that $\left\{B(\Pi): \Pi \in \mathcal{P}^{*}(G, A)\right\}$ is the set of bases of a matroid. The following lemma extends that result by providing an exchange property on all $A$-collections. The proof is essentially the same as the proof given in [1]. (For sets $A$ and $B$, we let $A \Delta B=(A-B) \cup(B-A)$.)

Lemma 3.1. Let $\Pi_{1}, \Pi_{2} \in \mathcal{P}(G, A)$ and let $p_{1} \in B\left(\Pi_{1}\right)-B\left(\Pi_{2}\right)$. Then there exists $\Pi_{1}^{\prime} \in \mathcal{P}(G, A)$ and $p_{2} \in B\left(\Pi_{1}\right) \cup B\left(\Pi_{2}\right)$ such that $B\left(\Pi_{1}^{\prime}\right)=$ $B\left(\Pi_{1}\right) \Delta\left\{p_{1}, p_{2}\right\}$. Moreover, given $\Pi_{1}, \Pi_{2}$, and $p_{1}$, we can find $\Pi_{1}^{\prime}$ and $p_{2}$ in $O\left(|V(G)|^{2}\right)$ time.

Proof. Let $\mathcal{B}=\{B(\Pi): \Pi \in \mathcal{P}(G, A)\}$. Suppose, by way of contradiction, that there exist 
3.1.1. $\Pi_{1}, \Pi_{2} \in \mathcal{P}(G, A)$ and $p_{1}=(u, \alpha) \in B\left(\Pi_{1}\right)-B\left(\Pi_{2}\right)$ such that $B\left(\Pi_{1}\right) \Delta\left\{p_{1}, p_{2}\right\} \notin \mathcal{B}$ for each $p_{2} \in B\left(\Pi_{1}\right) \cup B\left(\Pi_{2}\right)$.

Given an $A$-collection $\Pi$, we let $E(\Pi)$ denote the union of the edge sets $(E(P): P \in \Pi)$.

3.1.2. We choose $\Pi_{1}, \Pi_{2}$, and $p_{1}=(u, \alpha)$ satisfying 3.1 .1 with $\mid E\left(\Pi_{1}\right) \cup$ $E\left(\Pi_{2}\right) \mid$ as small as possible.

We use the following claim repeatedly.

3.1.3. There do not exist $\Pi_{1}^{\prime} \in \mathcal{P}(G, A)$ and $p_{2} \in V(G) \times \Gamma$ such that $B\left(\Pi_{1}^{\prime}\right)=$ $B\left(\Pi_{1}\right) \Delta\left\{p_{1}, p_{2}\right\}$ and $\left|E\left(\Pi_{1}^{\prime}\right) \cup E\left(\Pi_{2}\right)\right|<\left|E\left(\Pi_{1}\right) \cup E\left(\Pi_{2}\right)\right|$.

Proof of claim. Suppose otherwise. By 3.1.1, $p_{2} \notin B\left(\Pi_{1}\right) \cup B\left(\Pi_{2}\right)$. However, $\left|E\left(\Pi_{1}^{\prime}\right)-E\left(\Pi_{2}\right)\right|<\left|E\left(\Pi_{1}\right)-E\left(\Pi_{2}\right)\right|$. So, by 3.1.2, $\Pi_{1}^{\prime}, \Pi_{2}$, and $p_{2}$ do not satisfy 3.1.1. That is, there exists an element $p_{3} \in B\left(\Pi_{2}\right)-B\left(\Pi_{1}^{\prime}\right)$ such that $B\left(\Pi_{1}^{\prime}\right) \Delta\left\{p_{2}, p_{3}\right\} \in \mathcal{B}$. However, $B\left(\Pi_{1}\right) \Delta\left\{p_{1}, p_{3}\right\}=B\left(\Pi_{1}^{\prime}\right) \Delta\left\{p_{2}, p_{3}\right\} \in \mathcal{B}$, contradicting 3.1.1.

Let $p_{1}=(u, \alpha)$ and let $P=\left(v_{0}, e_{1}, v_{1}, \ldots, e_{k}, v_{k}\right)$ be the path in $\Pi_{1}$ ending at $u$; thus $P$ is loose. By possibly reversing the order, we may assume that there is a path $P^{\prime}=\left(v_{0}^{\prime}, e_{1}^{\prime}, v_{1}^{\prime}, \ldots, e_{l}^{\prime}, v_{l}^{\prime}\right)$ in $\Pi_{2}$ that starts at $v_{0}$. Suppose that $P$ is not contained in $P^{\prime}$. Now let $\Pi_{1}^{\prime}$ be the $A$-collection obtained from $\Pi_{1}$ by replacing $P$ with the trivial path $\left(v_{0}\right)$. Note that $B\left(\Pi_{1}^{\prime}\right)=B\left(\Pi_{1}\right) \Delta\left\{p_{1},\left(v_{0}, 0\right)\right\}$ and $\left|E\left(\Pi_{1}^{\prime}\right) \cup E\left(\Pi_{2}\right)\right|<\left|E\left(\Pi_{1}\right) \cup E\left(\Pi_{2}\right)\right|$, contradicting 3.1.3. Hence $P$ is contained in $P^{\prime}$.

Suppose that $P^{\prime}$ is disjoint from each path in $\Pi_{1}-\{P\}$ and let $\Pi_{1}^{\prime}$ be obtained from $\Pi_{1}$ by replacing $P$ with $P^{\prime}$. Note that $B\left(\Pi_{1}^{\prime}\right)=$ $B\left(\Pi_{1}\right) \Delta\left\{p_{1},\left(v_{l}^{\prime}, \omega_{G}\left(P^{\prime}\right)\right)\right\}$ and $\left(v_{l}^{\prime}, \omega_{G}\left(P^{\prime}\right)\right) \in B\left(\Pi_{2}\right)$, contradicting 3.1.1. Therefore there is some vertex that is both on $P^{\prime}$ and on a path in $\Pi_{1}$ other than $P$; let $v_{i}^{\prime}$ be the first such vertex on $P^{\prime}$ and let $Q=\left(u_{0}, f_{1}, u_{1}, \ldots, f_{m}, u_{m}\right)$ be the path of $\Pi_{1}$ containing $v_{i}^{\prime}$. Suppose that $u_{j}=v_{i}^{\prime}$.

For a walk $W=\left(x_{0}, f_{1}, x_{1}, \ldots, f_{p}, x_{p}\right)$ and $0 \leq a \leq b \leq p$ we denote the walks $\left(x_{a}, f_{a+1}, x_{a+1}, \ldots, f_{b}, x_{b}\right)$ and $\left(x_{b}, f_{b}, x_{b-1}, \ldots, f_{a+1}, x_{a}\right)$ by $W\left[x_{a}, x_{b}\right]$ and $W\left[x_{b}, x_{a}\right]$ respectively.

We consider two cases.

Case 1. $Q$ is a loose path.

Let $P_{1}$ be the $A$-path obtained by concatenating $P^{\prime}\left[v_{0}^{\prime}, \ldots, v_{i}^{\prime}\right]$ with $Q\left[u_{j}, \ldots, u_{0}\right]$ and let $P_{2}$ be the path obtained by concatenating $P^{\prime}\left[v_{0}^{\prime}, \ldots, v_{i}^{\prime}\right]$ with $Q\left[u_{j}, \ldots, u_{m}\right]$.

Case 1.1. $\omega_{G}\left(P_{1}\right) \neq 0$.

Let $\Pi_{1}^{\prime}=\left(\Pi_{1}-\{P, Q\}\right) \cup P_{1}$. Note that $B\left(\Pi_{1}^{\prime}\right)=B\left(\Pi_{1}\right)-\left\{p_{1},\left(u_{m}, \omega_{G}(Q)\right)\right\}$ and $\left(u_{m}, \omega_{G}(Q)\right) \in B\left(\Pi_{1}\right)$, contradicting 3.1.1. 
Case 1.2. $\omega_{G}\left(P_{1}\right)=0$.

Thus $\omega\left(P^{\prime}\left[v_{0}^{\prime}, v_{i}^{\prime}\right]\right)=\omega\left(Q\left[u_{0}, u_{j}\right]\right)$ and, hence, $\omega\left(P_{2}\right)=\omega(Q)$. Now let $\Pi_{1}^{\prime}$ be the $A$-collection obtained from $\Pi_{1}$ by replacing $P$ and $Q$ with $P_{2}$ and the trivial path $\left(u_{0}\right)$. Note that $B\left(\Pi_{1}^{\prime}\right)=\left(B\left(\Pi_{1}\right)-\left\{p_{1}\right\}\right) \cup\left\{\left(u_{0}, 0\right)\right\}$. Moreover, since $\omega_{G}\left(P_{1}\right)=0, P_{1} \neq P^{\prime}$. Thus there is an edge of $Q\left[u_{0}, u_{j}\right]$ that is not in $E\left(\Pi_{2}\right)$. So, $\left|E\left(\Pi_{1}^{\prime}\right) \cup E\left(\Pi_{2}\right)\right|<\left|E\left(\Pi_{1}\right) \cup E\left(\Pi_{2}\right)\right|$, contradicting 3.1.3.

Case 2. $Q$ is an A-path.

Let $P_{1}$ and $P_{2}$ be the $A$-paths in $G\left[E\left(P^{\prime}\right) \cup E(Q)\right]$ that both start at $v_{0}$ and that end with $u_{0}$ and $u_{m}$ respectively. Note that $\omega\left(P_{1}\right)+\omega(Q)-\omega\left(P_{2}\right)=0$ and $\omega(Q) \neq 0$, so either $\omega\left(P_{1}\right) \neq 0$ or $\omega\left(P_{2}\right) \neq 0$. Moreover, either $P^{\prime}$ is loose (and hence different from $P_{1}$ and $P_{2}$ ) or $\omega\left(P^{\prime}\right) \neq 0$. Thus either $\omega\left(P_{1}\right) \neq 0$ and $P_{2} \neq P^{\prime}$ or $\omega\left(P_{2}\right) \neq 0$ and $P_{1} \neq P^{\prime}$. By possibly swapping $P_{1}$ and $P_{2}$ and reversing $Q$, we may assume that $\omega\left(P_{2}\right) \neq 0$ and $P_{1} \neq P^{\prime}$. Let $\Pi_{1}^{\prime}$ be the $A$-collection obtained from $\Pi_{1}$ by replacing $P$ and $Q$ with $P_{2}$ and the trivial path $\left(u_{0}\right)$. Note that $B\left(\Pi_{1}^{\prime}\right)=\left(B\left(\Pi_{1}\right)-\left\{p_{1}\right\}\right) \cup\left\{\left(u_{0}, 0\right)\right\}$. Moreover, since $P_{1} \neq P^{\prime}$ there is an edge of $Q\left[u_{0}, u_{j}\right]$ that is not in $E\left(\Pi_{1}^{\prime}\right) \cup E\left(\Pi_{2}\right)$. Thus $\left|E\left(\Pi_{1}^{\prime}\right) \cup E\left(\Pi_{2}\right)\right|<\left|E\left(\Pi_{1}\right) \cup E\left(\Pi_{2}\right)\right|$, contradicting 3.1.3. This final contradiction completes the proof.

The above proof can easily be made algorithmic with the stated running time.

We now prove a useful application of the exchange property.

Lemma 3.2. Let $\Pi_{1}, \Pi_{2} \in \mathcal{P}(G, A)$ and let $B_{1} \subseteq B_{A}\left(\Pi_{1}\right)$. Then there exists $\Pi_{3} \in \mathcal{P}(G, A)$ such that either:

(1) $\operatorname{val}\left(\Pi_{3}\right)=\operatorname{val}\left(\Pi_{1}\right)$ and $B_{1} \subseteq B\left(\Pi_{3}\right)$ and $B\left(\Pi_{3}\right)-B_{1} \subseteq B\left(\Pi_{2}\right)$, or

(2) $\operatorname{val}\left(\Pi_{3}\right)=\operatorname{val}\left(\Pi_{1}\right)+1$ and $\left|B\left(\Pi_{3}\right) \cap B_{1}\right| \geq\left|B_{1}\right|-1$.

Moreover, we can find such $\Pi_{3}$ in $O\left(|V(G)|^{3}\right)$ time.

Proof. We assume that:

3.2.1. Among all $\Pi_{1}^{\prime} \in \mathcal{P}(G, A)$ with $B_{1} \subseteq B\left(\Pi_{1}^{\prime}\right)$ and $\operatorname{val}\left(\Pi_{1}^{\prime}\right)=\operatorname{val}\left(\Pi_{1}\right)$ we choose $\Pi_{1}^{\prime}$ minimizing $\left|B\left(\Pi_{1}^{\prime}\right)-B\left(\Pi_{2}\right)\right|$.

We may assume that there exists $p_{1} \in B\left(\Pi_{1}^{\prime}\right)-\left(B_{1} \cup B\left(\Pi_{2}\right)\right)$, since otherwise $\Pi_{3}:=\Pi_{1}^{\prime}$ satisfies (1). By the exchange property, there exists $\Pi_{3} \in \mathcal{P}(G, A)$ and $p_{2} \in B\left(\Pi_{1}^{\prime}\right) \cup B\left(\Pi_{2}\right)$ such that $B\left(\Pi_{3}\right)=B\left(\Pi_{1}^{\prime}\right) \Delta\left\{p_{1}, p_{2}\right\}$.

Case 1. $p_{2} \in B\left(\Pi_{1}^{\prime}\right)$.

Thus $\operatorname{val}\left(\Pi_{3}\right)=\operatorname{val}\left(\Pi_{1}^{\prime}\right)+1$ and $\left|B\left(\Pi_{3}\right) \cap B_{1}\right| \geq\left|B_{1}\right|-1$, satisfying (2). 
Case 2. $p_{2} \in B\left(\Pi_{2}\right)-B\left(\Pi_{1}^{\prime}\right)$.

Thus val $\left(\Pi_{3}\right)=\operatorname{val}\left(\Pi_{1}^{\prime}\right), B_{1} \subseteq B\left(\Pi_{3}\right)$, and $\left|B\left(\Pi_{3}\right)-B\left(\Pi_{2}\right)\right|<\mid B\left(\Pi_{1}^{\prime}\right)-$ $B\left(\Pi_{2}\right) \mid$, contradicting 3.2.1.

That completes the proof; this proof can clearly be made algorithmic with the stated running time.

The following two lemmas are consequences of Lemma 3.2.

Lemma 3.3. Let $\Pi_{1}, \Pi_{2} \in \mathcal{P}(G, A)$ with $\operatorname{val}\left(\Pi_{2}\right)=\operatorname{val}\left(\Pi_{1}\right)+1$, let $u v=e \in$ $E(G)$, let $(u, \alpha)$ and $p$ be distinct elements of $B\left(\Pi_{1}\right)$, and let $(v, \beta) \in B\left(\Pi_{2}\right)$ where $\alpha+\omega_{G}(e, v)-\beta \neq 0$. Then there exists $\Pi_{3} \in \mathcal{P}(G, A)$ such that $\operatorname{val}\left(\Pi_{3}\right)=$ $\operatorname{val}\left(\Pi_{2}\right)$ and either $(u, \alpha) \in B\left(\Pi_{3}\right)$ or $p \in B\left(\Pi_{3}\right)$. Moreover, we can find such $\Pi_{3}$ in $O\left(|V(G)|^{3}\right)$ time.

Proof. By Lemma 3.2 with $B_{1}=\{p,(u, \alpha)\}$, we get one of the following two cases.

Case 1. There exists $\Pi \in \mathcal{P}(G, A)$ such that $\operatorname{val}(\Pi)=\operatorname{val}\left(\Pi_{1}\right)$ and $B_{1} \subseteq$ $B(\Pi)$ and $B(\Pi)-B_{1} \subseteq B\left(\Pi_{2}\right)$.

Since $|B(\Pi)|=2 \operatorname{val}(\Pi)=2 \operatorname{val}\left(\Pi_{2}\right)+2=\left|B\left(\Pi_{2}\right)\right|+\left|B_{1}\right|$ and $B(\Pi)-B_{1} \subseteq$ $B\left(\Pi_{2}\right)$, we have $B\left(\Pi_{2}\right) \subseteq B(\Pi)$. Thus $p,(u, \alpha),(v, \beta) \in B(\Pi)$. Let $P_{u}$ and $P_{v}$ be the loose paths in $\Pi$ ending at $u$ and $v$ respectively. Now let $P=$ $\left(P_{u}, e, \bar{P}_{v}\right)$, where $\bar{P}_{v}$ denotes the reverse of the path $P_{v}$. Note that $P$ is an $A$-path and $\omega_{G}(P)=\alpha+\omega_{G}(e, v)-\beta \neq 0$. Now let $\Pi_{3}=\left(\Pi-\left\{P_{u}, P_{v}\right\}\right) \cup\{P\}$. Note that $\operatorname{val}_{A}\left(\Pi_{3}\right)=\operatorname{val}\left(\Pi_{2}\right)$ and $p \in B\left(\Pi_{3}\right)$, as required.

Case 2. There exists $\Pi_{3} \in \mathcal{P}(G, A)$ such that $\operatorname{val}\left(\Pi_{3}\right)=\operatorname{val}\left(\Pi_{2}\right)$ and $\mid B\left(\Pi_{3}\right) \cap$ $B_{1}|\geq| B_{1} \mid-1$.

Thus either $(u, \alpha) \in B\left(\Pi_{3}\right)$ or $p \in B\left(\Pi_{3}\right)$, as required.

This proof is clearly constructive with the stated running time.

The next lemma is a direct consequence of Lemma 3.2; we omit the easy proof.

Lemma 3.4. Let $\Pi_{1}, \Pi_{2} \in \mathcal{P}(G, A)$ with $\operatorname{val}\left(\Pi_{2}\right)=\operatorname{val}\left(\Pi_{1}\right)$, let $p_{1} \in B\left(\Pi_{1}\right)$, and let $p_{2}$ and $p_{3}$ be distinct elements of $B\left(\Pi_{2}\right)$. Then there exists $\Pi_{3} \in$ $\mathcal{P}(G, A)$ such that either:

(1) $\operatorname{val}\left(\Pi_{3}\right)=\operatorname{val}\left(\Pi_{1}\right), p_{1} \in B\left(\Pi_{3}\right)$, and either $p_{2} \in B\left(\Pi_{3}\right)$ or $p_{3} \in B\left(\Pi_{3}\right)$, or

(2) $\operatorname{val}\left(\Pi_{3}\right)=\operatorname{val}\left(\Pi_{1}\right)+1$.

Moreover, we can find such $\Pi_{3}$ in $O\left(|V(G)|^{3}\right)$ time. 


\section{Key lemmas}

In this section we prove constructive analogues of some of the lemmas in Section 2.

Throughout this section we let $G$ be a $\Gamma$-labelled graph, $A \subseteq V(G)$, and $\mathcal{P} \subseteq \mathcal{P}(G, A)$. We use the following definitions:

$$
\begin{aligned}
\nu(\mathcal{P}, A) & =\max \left(\operatorname{val}_{A}(\Pi): \Pi \in \mathcal{P}\right), \\
\operatorname{def}(\mathcal{P}, A) & =|A|-2 \nu(\mathcal{P}, A), \\
\mathcal{P}^{*} & =\left\{\Pi \in \mathcal{P}: \operatorname{val}_{A}(\Pi)=\nu(\mathcal{P}, A)\right\}, \text { and } \\
\mathcal{R}(\mathcal{P}, A) & =\cup\left(B_{A}(\Pi): \Pi \in \mathcal{P}^{*}\right) .
\end{aligned}
$$

Now, for each $v \in V(G)$, we let

$$
\Gamma(\mathcal{P}, A, v)=\{\gamma \in \Gamma:(v, \gamma) \in \mathcal{R}(\mathcal{P}, A)\} .
$$

In addition, we define:

$$
\begin{aligned}
D_{1}(\mathcal{P}, A) & =\{v \in V(G):|\Gamma(\mathcal{P}, A, v)|=1\}, \\
D_{2}(\mathcal{P}, A) & =\{v \in V(G):|\Gamma(\mathcal{P}, A, v)|>1\}, \text { and } \\
D(\mathcal{P}, A) & =D_{1}(\mathcal{P}, A) \cup D_{2}(\mathcal{P}, A) .
\end{aligned}
$$

We begin with some easy observations relating to 2.1 and 2.2 :

4.1. Let $u \in A-D(\mathcal{P}, A)$. If there exists $\Pi \in \mathcal{P}(G-u, A-\{u\})$ such that $\operatorname{val}_{A-\{u\}}(\Pi)=\nu(\mathcal{P}, A)$, then there exists $\Pi^{\prime} \in \mathcal{P}(G, A)$ such that $(u, 0) \in$ $\mathcal{R}\left(\mathcal{P} \cup\left\{\Pi^{\prime}\right\}, A\right)$.

4.2. Let $u \in V(G)-A$ with $\Gamma(\mathcal{P}, A, u) \subseteq\{0\}$. If there exists $\Pi \in \mathcal{P}(G, A \cup\{u\})$ such that $\operatorname{val}_{A \cup\{u\}}(\Pi)=\nu(\mathcal{P}, A)+1$, then there exists $\Pi^{\prime} \in \mathcal{P}(G, A)$ such that either $\operatorname{val}_{A}\left(\Pi^{\prime}\right)>\nu(\mathcal{P}, A)$ or $\operatorname{val}_{A}\left(\Pi^{\prime}\right)=\nu(\mathcal{P}, A)$ and there exists $\alpha \in \Gamma-\{0\}$ such that $(u, \alpha) \in \mathcal{R}\left(\mathcal{P} \cup\left\{\Pi^{\prime}\right\}, A\right)$.

The next result generalizes Lemma 2.3.

Lemma 4.3. Let $u \in A-D(\mathcal{P}, A)$, $u v=e \in E(G)$, and $\alpha \in \Gamma(\mathcal{P}, A, v)$ such that $\omega_{G}(e, u) \neq-\alpha$. If $\Pi \in \mathcal{P}(G-u, A-\{u\})$ with $\operatorname{val}_{A-\{u\}}(\Pi)=$ $\nu(\mathcal{P}, A)-1$ and $p \in B_{A-\{u\}}(\Pi)-\mathcal{R}(\mathcal{P}, A)$, then there exists $\Pi^{\prime} \in \mathcal{P}(G, A)$ such that $\operatorname{val}_{A}\left(\Pi^{\prime}\right)=\nu(\mathcal{P}, A)$ and either $\left(u, \alpha+\omega_{G}(e, u)\right) \in B\left(\Pi^{\prime}\right)$ or $p \in B\left(\Pi^{\prime}\right)$. Moreover, if $|\mathcal{P}| \leq 2|V(G)|$, then we can find such $\Pi^{\prime}$ in $O\left(|V(G)|^{3}\right)$ time. 
Proof. Let $\Pi_{1}$ be the $A$-collection obtained by adding the trivial path $(u)$ to $\Pi$. Note that $\operatorname{val}_{A}\left(\Pi_{1}\right)=\nu(\mathcal{P}, A)-1$ and $p,(u, 0) \in B_{A}\left(\Pi_{1}\right)$. Let $\Pi_{2} \in \mathcal{P}^{*}$ with $(v, \alpha) \in B_{A}\left(\Pi_{2}\right)$. Now $\operatorname{val}_{A}\left(\Pi_{2}\right)=\operatorname{val}_{A}\left(\Pi_{1}\right)+1$. Therefore, by Lemma 3.3, we find $\Pi^{\prime} \in \mathcal{P}(G, A)$ with $\operatorname{val}_{A}\left(\Pi^{\prime}\right)=\nu(\mathcal{P}, A)$ and either $(u, 0) \in B_{A}\left(\Pi^{\prime}\right)$ or $p \in B_{A}\left(\Pi^{\prime}\right)$. Now $\Pi^{\prime}$ satisfies the lemma.

This proof is clearly constructive with the stated running time.

The next result generalizes Lemma 2.4.

Lemma 4.4. Let $u \in V(G)-A$ with $\Gamma(\mathcal{P}, A, u) \subseteq\{0\}$, let $u v=e \in E(G)$ with $\omega_{G}(e, v) \in \Gamma(\mathcal{P}, A, v)$. If $\Pi \in \mathcal{P}(G, A \cup\{u\})$ with $\operatorname{val}_{A \cup\{u\}}(\Pi)=\nu(\mathcal{P}, A)$ and $p \in B_{A \cup\{u\}}(\Pi)-\mathcal{R}(\mathcal{P}, A)$, then there exists $\Pi^{\prime} \in \mathcal{P}(G, A)$ such that either $\operatorname{val}_{A}\left(\Pi^{\prime}\right)>\nu(\mathcal{P}, A)$ or $\operatorname{val}_{A}\left(\Pi^{\prime}\right)=\nu(\mathcal{P}, A)$ and either $p \in B\left(\Pi^{\prime}\right)$ or there exists $(u, \alpha) \in B\left(\Pi^{\prime}\right)$ with $\alpha \neq 0$. Moreover, if $|\mathcal{P}| \leq 2|V(G)|$, then we can find such $\Pi^{\prime}$ in $O\left(|V(G)|^{3}\right)$ time.

Proof. Note that, if $v \in A$, then, since $\omega_{G}(e, v) \in \Gamma(\mathcal{P}, A, v)$, we have $\omega_{G}(e, v)=0$. On the other hand, if $v \notin A$, then, by possibly shifting, we may assume that $\omega_{G}(e, v)=0$. Let $p=(w, \delta)$. We break the proof into the following cases.

Case 1. There exists $\Pi_{1} \in \mathcal{P}(G, A \cup\{u\})$ with $\operatorname{val}_{A \cup\{u\}}\left(\Pi_{1}\right)=\nu(\mathcal{P}, A)$ and $p \in$ $B_{A \cup\{u\}}\left(\Pi_{1}\right)$, such that $u$ is not the start of the loose path in $\Pi_{1}$ containing $w$.

There is a path $P \in \Pi_{1}$ whose start or end is $u$. Suppose that $P$ is a loose path with respect to $A \cup\{u\}$; thus $u$ is the start of $P$ and $P$ does not contain $w$. Then $\Pi^{\prime}:=\Pi_{1}-\{P\}$ satisfies the lemma. Therefore we may assume that $P$ is an $A \cup\{u\}$-path; furthermore, by possibly reversing $P$, we may assume that $u$ is the end of $P$. Let $\alpha=\omega_{G}(P)$. Since $P$ is an $A \cup\{u\}$-path in $\Pi_{1}$, we have $\alpha \neq 0$. Now note that $\Pi_{1} \in \mathcal{P}(G, A), \operatorname{val}_{A}\left(\Pi_{1}\right)=\nu(\mathcal{P}, A)-1$, and $p,(u, \alpha) \in B_{A}\left(\Pi_{1}\right)$. Let $\Pi_{2} \in \mathcal{P}^{*}$ with $(v, 0) \in B_{A}\left(\Pi_{2}\right)$. Applying Lemma 3.3 to $\Pi_{1}$ and $\Pi_{2}$ we find $\Pi^{\prime} \in \mathcal{P}(G, A)$ with $\operatorname{val}_{A}\left(\Pi^{\prime}\right)=\nu(\mathcal{P}, A)$ and either $p \in B_{A}\left(\Pi^{\prime}\right)$ or $(u, \alpha) \in B_{A}\left(\Pi^{\prime}\right)$, as required by the lemma.

Case 2. There exists $\Pi_{1} \in \mathcal{P}(G, A \cup\{u\})$ with $\operatorname{val}_{A \cup\{u\}}(\Pi)=\nu(\mathcal{P}, A)+1$.

There is a path $P \in \Pi_{1}$ whose start or end is $u$. If $P$ is a loose path, then $\Pi^{\prime}:=\Pi_{1}-\{P\}$ satisfies the lemma. Therefore we may assume that $P$ is an $A \cup\{u\}$-path; furthermore, by possibly reversing $P$, we may assume that $u$ is the end of $P$. Let $\alpha=\omega_{G}(P)$. Since $P$ is an $A \cup\{u\}$-path in $\Pi_{1}$, we have $\alpha \neq 0$. Now note that $\Pi_{1}$ is an $A$-collection, $\operatorname{val}_{A}\left(\Pi_{1}\right)=\nu(\mathcal{P}, A)$, and $(u, \alpha) \in B_{A}\left(\Pi_{1}\right)$. Thus $\Pi^{\prime}:=\Pi_{1}$ satisfies the lemma.

Case 3. There exists $\Pi_{1} \in \mathcal{P}(G, A \cup\{u\})$ with $\operatorname{val}_{A \cup\{u\}}\left(\Pi_{1}\right)=\nu(\mathcal{P}, A)$ and there exists $(z, \beta) \in B_{A \cup\{u\}}\left(\Pi_{1}\right)-\{(w, \delta)\}$ with $z u=f \in E(G)$. 
Let $P \in \Pi_{1}$ be the path ending at $w$. We may assume that $u$ is the start of $P$, since otherwise we reduce to Case 1 . Let $P_{z} \in \Pi_{1}$ be the path ending at $z$, let $P_{u}=\left(P_{z}, f, u\right)$, and let $P_{w}=\left(P_{z}, f, P\right)$. Let $\alpha=\omega_{G}\left(P_{u}\right)$. Note that $\omega_{G}\left(P_{w}\right)=\alpha+\delta$, so either $\alpha \neq 0$ or $\omega_{G}\left(P_{w}\right)=\delta$. Suppose that $\alpha \neq 0$. Let $\Pi^{\prime}=\left(\Pi_{1}-\left\{P, P_{z}\right\}\right) \cup\left\{P_{u}\right\}$. Note that $\operatorname{val}_{A}\left(\Pi^{\prime}\right)=\nu(\mathcal{P}, A)$ and $(u, \alpha) \in B_{A}\left(\Pi^{\prime}\right)$, as required. Now suppose that $\omega_{G}\left(P_{w}\right)=\delta$. Let $\Pi^{\prime}=\left(\Pi_{1}-\left\{P, P_{z}\right\}\right) \cup\left\{P_{w}\right\}$. Note that $\operatorname{val}_{A}\left(\Pi^{\prime}\right)=\nu(\mathcal{P}, A)$ and $(w, \delta) \in B_{A}\left(\Pi^{\prime}\right)$, as required.

Case 4. There exists $\Pi_{2} \in \mathcal{P}(G, A \cup\{u\})$ such that $\operatorname{val}_{A \cup\{u\}}\left(\Pi_{2}\right)=$ $\nu(\mathcal{P}, A)$ and $(z, \beta),(v, 0) \in B_{A \cup\{u\}}\left(\Pi_{2}\right)$ where $z u=f \in E(G)$ and $(z, \beta) \notin$ $\{(w, \delta),(v, 0)\}$.

Note that, since $(v, 0) \in \mathcal{R}(\mathcal{P}, A)$, we have $(v, 0) \neq(w, \delta)$. Recall that $\Pi \in \mathcal{P}(G, A \cup\{u\}), \operatorname{val}_{A \cup\{u\}}(\Pi)=\nu(\mathcal{P}, A)$, and $(w, \delta) \in B_{A \cup\{u\}}(\Pi)$. Applying Lemma 3.4 to $\Pi_{1}:=\Pi$ and $\Pi_{2}$, we find $\Pi_{3} \in \mathcal{P}(G, A)$ such that either $\operatorname{val}_{A \cup\{u\}}\left(\Pi_{3}\right)>\nu(\mathcal{P}, A)$, or $\operatorname{val}_{A \cup\{u\}}\left(\Pi_{3}\right)=\nu(\mathcal{P}, A)$ and either $(v, 0),(w, \delta) \in$ $B_{A \cup\{u\}}\left(\Pi_{3}\right)$ or $(z, \beta),(w, \delta) \in B_{A \cup\{u\}}\left(\Pi_{3}\right)$. The case that $\operatorname{val}_{A \cup\{u\}}\left(\Pi_{3}\right)>$ $\nu(\mathcal{P}, A)$ reduces to Case 2 and the case that $\operatorname{val}_{A \cup\{u\}}\left(\Pi_{3}\right)=\nu(\mathcal{P}, A)$ and either $(v, 0),(w, \delta) \in B_{A \cup\{u\}}\left(\Pi_{3}\right)$ or $(z, \beta),(w, \delta) \in B_{A \cup\{u\}}\left(\Pi_{3}\right)$ reduces to Case 3.

Case 5. There exists $\Pi_{2} \in \mathcal{P}(G, A \cup\{u\})$ such that $\operatorname{val}_{A \cup\{u\}}\left(\Pi_{2}\right)=\nu(\mathcal{P}, A)$ and $(u, 0),(v, 0) \in B_{A \cup\{u\}}\left(\Pi_{2}\right)$.

Note that, since $(v, 0) \in \mathcal{R}(\mathcal{P}, A)$, we have $(v, 0) \neq(w, \delta)$. Recall that $\Pi \in \mathcal{P}(G, A \cup\{u\}), \operatorname{val}_{A \cup\{u\}}(\Pi)=\nu(\mathcal{P}, A)$, and $(w, \delta) \in B_{A \cup\{u\}}(\Pi)$. Applying Lemma 3.4 to $\Pi_{1}:=\Pi$ and $\Pi_{2}$, we find $\Pi_{3} \in \mathcal{P}(G, A)$ such that either $\operatorname{val}_{A \cup\{u\}}\left(\Pi_{3}\right)>\nu(\mathcal{P}, A)$, or $\operatorname{val}_{A \cup\{u\}}\left(\Pi_{3}\right)=\nu(\mathcal{P}, A)$ and either $(u, 0),(w, \delta) \in B_{A \cup\{u\}}\left(\Pi_{3}\right)$ or $(v, 0),(w, \delta) \in B_{A \cup\{u\}}\left(\Pi_{3}\right)$. The case that $\operatorname{val}_{A \cup\{u\}}\left(\Pi_{3}\right)>\nu(\mathcal{P}, A)$ reduces to Case 2 ; the case that $\operatorname{val}_{A \cup\{u\}}\left(\Pi_{3}\right)=$ $\nu(\mathcal{P}, A)$ and $(v, 0),(w, \delta) \in B_{A \cup\{u\}}\left(\Pi_{3}\right)$ reduces to Case 3 ; and the case that $\operatorname{val}_{A \cup\{u\}}\left(\Pi_{3}\right)=\nu(\mathcal{P}, A)$ and $(u, 0),(w, \delta) \in B_{A \cup\{u\}}\left(\Pi_{3}\right)$ reduces to Case 1.

Let $\Pi_{v} \in \mathcal{P}^{*}$ with $(v, 0) \in B_{A}\left(\Pi_{v}\right)$. We may assume that there is a path $P \in \Pi_{v}$ that contains $u$, since otherwise $\Pi_{2}:=\Pi_{v} \cup\{(u)\}$ meets the criteria of Case 5.

Case 6. $P$ is a loose path with respect to $A$.

For any $y \in V(P)$, we let $P_{y}$ denote the initial segment of $P$ ending at $y$. We may assume that $\omega_{G}\left(P_{u}\right)=0$, since otherwise $\Pi^{\prime}:=\left(\Pi_{v}-\{P\}\right) \cup\left\{P_{u}\right\}$ satisfies the lemma. Now we may assume that $v$ is the end of $P$, since otherwise $\Pi_{2}:=\left(\Pi_{v}-\{P\}\right) \cup\left\{P_{u}\right\}$ meets the criteria of Case 5 . Now let $z$ be the vertex preceding $u$ on $P$ and let $P^{\prime}$ be the subpath of $P$ starting at $u$ and ending at $v$. Let $\beta=\omega_{G}\left(P_{z}\right)$. We may assume that $(z, \beta) \neq(w, \delta)$, since 
otherwise $\Pi^{\prime}:=\left(\Pi_{v}-\{P\}\right) \cup\left\{P_{z}\right\}$ satisfies the lemma. Finally, we see that $\Pi_{2}:=\left(\Pi_{v}-\{P\}\right) \cup\left\{P_{z}, P^{\prime}\right\}$ meets the criteria of Case 4 .

Case 7. $P$ is an A-path.

For any $y \in V(P)$, we let $P_{y}$ denote the initial segment of $P$ ending at $y$. Note that, by possibly reversing the direction of $P$, we may assume that $\omega_{G}\left(P_{u}\right) \neq 0$; let $\alpha=\omega_{G}\left(P_{u}\right)$. Let $z$ be the vertex on $P$ immediately following $u$, let $P^{\prime}$ denote the subpath of $\bar{P}$ that ends at $z$, and let $\beta=\omega_{G}\left(P^{\prime}\right)$. We may assume that $(z, \beta)=(w, \delta)$, since otherwise $\Pi_{2}:=\left(\Pi_{v}-\{P\}\right) \cup\left\{P_{u}, P^{\prime}\right\}$ meets the criteria of Case 4 . Let $Q \in \Pi_{v}$ be the path ending at $v$. Let $P^{\prime \prime}=\left(Q, e, \bar{P}_{u}\right)$. Note that $P^{\prime \prime}$ is an $A$-path and that $\omega_{G}\left(P^{\prime \prime}\right)=\omega_{G}(Q)+\omega_{G}(e, u)-\omega_{G}\left(P_{u}\right)=$ $-\alpha \neq 0$. Therefore $\Pi^{\prime}:=\left(\Pi_{v}-\{P, Q\}\right) \cup\left\{P^{\prime}, P^{\prime \prime}\right\}$ satisfies the lemma.

That completes the proof; this proof can easily be made algorithmic with the stated running time.

We say that $(G, A)$ is $\mathcal{P}$-critical if $G$ is connected, $E_{0}(G, A)=\emptyset$, $D_{1}(\mathcal{P}, A)=A$, and $D_{2}(\mathcal{P}, A)=V(G)-A$. The next result generalizes Lemma 2.7.

Lemma 4.5. If $(G, A)$ is $\mathcal{P}$-critical and $\operatorname{def}(\mathcal{P}, A)>1$, then there exists $\Pi \in \mathcal{P}(G, A)$ such that $\operatorname{val}_{A}(G)=\nu_{A}(\mathcal{P})+1$. Moreover, if $|\mathcal{P}| \leq 2|V(G)|$, then we can find such $\Pi^{\prime}$ in $O\left(|V(G)|^{4}\right)$ time.

Proof. We start by considering an easy case.

Case 1. There exists $\Pi_{1} \in \mathcal{P}(G, A)$ with $\operatorname{val}_{A}\left(\Pi_{1}\right)=\nu_{A}(\mathcal{P})$ and there exists $(u, \alpha),(v, \beta) \in B_{A}\left(\Pi_{1}\right)$ where $u v=e \in E(G)$.

We break this into two further subcases.

Case 1.1. $\alpha+\omega_{G}(e, v)-\beta \neq 0$.

Let $P_{u}, P_{v} \in \Pi_{1}$ be the paths ending at $u$ and $v$ respectively and let $P=\left(P_{u}, e, \bar{P}_{v}\right)$. Note that $P$ is an $A$-path and that $\omega_{G}(P)=\alpha+\omega_{G}(e, v)-\beta \neq 0$. Thus $\Pi:=\left(\Pi_{1}-\left\{P_{u}, P_{v}\right\}\right) \cup\{P\}$ satisfies the lemma.

Case 1.2. $\alpha+\omega_{G}(e, v)-\beta=0$.

Note that, since $(G, A)$ is $\mathcal{P}$-critical, either $u \notin A$ or $v \notin A$. By possibly swapping $u$ and $v$, we may assume that $v \notin A$. Then, since $(G, A)$ is $\mathcal{P}_{\text {- }}$ critical, there exists $\beta^{\prime} \in \Gamma(\mathcal{P}, A, v)-\{\beta\}$. Let $\Pi_{2} \in \mathcal{P}^{*}$ with $\left(v, \beta^{\prime}\right) \in B_{A}\left(\Pi_{2}\right)$. Applying Lemma 3.4 to $\Pi_{2}$ and $\Pi_{1}$, we find $\Pi_{3} \in \mathcal{P}(G, A)$ such that either $\operatorname{val}_{A}\left(\Pi_{3}\right)>\nu_{A}(\mathcal{P})$ or $\operatorname{val}_{A}\left(\Pi_{3}\right)=\nu_{A}(\mathcal{P})$ and either $(u, \alpha),\left(v, \beta^{\prime}\right) \in B_{A}\left(\Pi_{3}\right)$ or $(v, \beta),\left(v, \beta^{\prime}\right) \in B_{A}\left(\Pi_{3}\right)$. If $\operatorname{val}_{A}\left(\Pi_{3}\right)>\nu_{A}(\mathcal{P})$, then $\Pi:=\Pi_{3}$ satisfies the lemma. Also, note that $B_{A}\left(\Pi_{3}\right)$ cannot contain both $(v, \beta)$ and $\left(v, \beta^{\prime}\right)$. Therefore we may assume that $(u, \alpha),\left(v, \beta^{\prime}\right) \in B_{A}\left(\Pi_{3}\right)$. Now, since $\beta \neq \beta^{\prime}$, we have $\alpha+\omega_{G}(e, v)-\beta^{\prime} \neq \alpha+\omega_{G}(e, v)-\beta=0$. Therefore $\Pi_{1}:=\Pi_{3}$ satisfies the criterion for Case 1.1. 
(*) Among all triples $\left(\Pi_{1},\left(v_{1}, \alpha_{1}\right),\left(v_{2}, \alpha_{2}\right)\right)$ where $\Pi_{1} \in \mathcal{P}(G, A), \operatorname{val}_{A}\left(\Pi_{1}\right)=$ $\nu_{A}(\mathcal{P})$, and $\left(v_{1}, \alpha_{1}\right),\left(v_{2}, \alpha_{2}\right) \in B_{A}\left(\Pi_{1}\right)$ we choose the triple such that the distance between $v_{1}$ and $v_{2}$ in $G$ is minimum.

In view of Case 1 , we may assume that $v_{1}$ is not adjacent to $v_{2}$. Let $P$ be a shortest $\left(v_{1}, v_{2}\right)$-path and let $u$ be an internal vertex of $P$. Since $(G, A)$ is $\mathcal{P}$-critical, there exists $\beta \in \Gamma(\mathcal{P}, A, u)$. Let $\Pi_{2} \in \mathcal{P}^{*}$ with $(u, \beta) \in B_{A}\left(\Pi_{2}\right)$. Applying Lemma 3.4 to $\Pi_{2}$ and $\Pi_{1}$, we find $\Pi_{3} \in \mathcal{P}(G, A)$ such that either $\operatorname{val}_{A}\left(\Pi_{3}\right)>\nu_{A}(\mathcal{P})$ or $\operatorname{val}_{A}\left(\Pi_{3}\right)=\nu_{A}(\mathcal{P})$ and $(u, \beta),\left(v_{i}, \alpha_{i}\right) \in B_{A}\left(\Pi_{3}\right)$ for some $i \in\{1,2\}$. If $\operatorname{val}_{A}\left(\Pi_{3}\right)>\nu_{A}(\mathcal{P})$, then $\Pi:=\Pi_{3}$ satisfies the lemma. Thus, by symmetry, we may assume that $\operatorname{val}_{A}\left(\Pi_{3}\right)=\nu_{A}(\mathcal{P})$ and $(u, \beta),\left(v_{1}, \alpha_{1}\right) \in B_{A}\left(\Pi_{3}\right)$. However, since $v_{1}$ is closer to $u$ than it is to $v_{2}$, we have a contradiction to $(*)$.

That completes the proof; this proof can easily be made algorithmic with the stated running time.

\section{The algorithm}

Throughout the algorithm we maintain a set $\mathcal{P} \subseteq \mathcal{P}(G, A)$. We are primarily interested in the sets $D_{1}(\mathcal{P}, A)$ and $D_{2}(\mathcal{P}, A)$. Therefore, by removing unnecessary $A$-collections from $\mathcal{P}$, we keep

$$
|\mathcal{P}| \leq\left|D_{1}(\mathcal{P}, A)\right|+2\left|D_{2}(\mathcal{P}, A)\right| \leq 2|V(G)| .
$$

If $\mathcal{P}_{1}, \mathcal{P}_{2} \subseteq \mathcal{P}(G, A)$, then we say that $\mathcal{P}_{2}$ is richer than $\mathcal{P}_{1}$, with respect to $A$, if either $\nu_{A}\left(\mathcal{P}_{2}\right)>\nu_{A}\left(\mathcal{P}_{1}\right)$ or $\nu_{A}\left(\mathcal{P}_{2}\right)=\nu_{A}\left(\mathcal{P}_{1}\right)$ and $\left|D_{1}\left(\mathcal{P}_{2}, A\right)\right|+2\left|D_{2}\left(\mathcal{P}_{2}, A\right)\right|>$ $\left|D_{1}\left(\mathcal{P}_{1}, A\right)\right|+2\left|D_{2}\left(\mathcal{P}_{1}, A\right)\right|$.

By possibly shifting (as we did in Lemma 1.2), we may assume that $(G, A)$ satisfies:

(1) for each $v \in D_{1}(\mathcal{P}, A), \Gamma(\mathcal{P}, A, v)=\{0\}$, and

(2) for each $u \in N_{G}(D(\mathcal{P}, A))-A$, there exists $u v=e \in E(G)$ such that $\omega_{G}(e, v) \in \Gamma(G, A, v)$.

Now let $A^{\prime}=A \cup N_{G}(D(G, A)) \cup D_{1}(G, A)$ and $X=N_{G-E_{0}\left(G, A^{\prime}\right)}(D(G, A))$.

Optimality condition. If $\operatorname{def}(\mathcal{P}, A)=\operatorname{odd}\left(G-X, A^{\prime}-X\right)-\left|A^{\prime}-A\right|-|X|$, then the $A$-collections in $\mathcal{P}^{*}$ are optimal.

Proof. Note that $\operatorname{def}(\mathcal{P}, A) \geq \operatorname{def}(G, A) \geq \operatorname{odd}\left(G-X, A^{\prime}-X\right)-\left|A^{\prime}-A\right|-|X|$. Thus, if $\operatorname{def}(\mathcal{P}, A)=\operatorname{odd}\left(G-X, A^{\prime}-X\right)-\left|A^{\prime}-A\right|-|X|$, then $\operatorname{def}(\mathcal{P}, A)=$ $\operatorname{def}(G, A)$ and, hence, each $A$-collection in $\mathcal{P}^{*}$ is optimal.

In each iteration of the algorithm, if $\operatorname{def}(\mathcal{P}, A) \neq \operatorname{odd}\left(G-X, A^{\prime}-X\right)-\mid A^{\prime}-$ $A|-| X \mid$, then we find an $A$-collection $\Pi$ such that $\mathcal{P} \cup\{\Pi\}$ is richer than $\mathcal{P}$. 
Hence in at most $O\left(|V(G)|^{2}\right)$ iterations we will find an optimal $A$-collection. It remains to show how we find the promised $A$-collection $\Pi$.

We omit the elementary proof of the next lemma.

Lemma 5.1. Let $A_{1}, X_{1} \subseteq V(G)$ such that $A \cup X_{1} \subseteq A_{1} \subseteq A^{\prime}$ and $X_{1} \subseteq X$. Then, in $O\left(|V(G)|^{3}\right)$ time, we can construct $\mathcal{P}_{1} \subset \mathcal{P}\left(G-X_{1}, A_{1}-X_{1}\right)$ such that either $\nu_{A_{1}}\left(\mathcal{P}_{1}\right)>\nu_{A}(\mathcal{P})-\left|X_{1}\right|$ or $\nu_{A_{1}}\left(\mathcal{P}_{1}\right)=\nu_{A}(\mathcal{P})-\left|X_{1}\right|$ and $\mathcal{R}(\mathcal{P}, A) \subseteq$ $\mathcal{R}\left(\mathcal{P}_{1}, A_{1}\right)$.

Lemma 5.2. Let $A^{\prime \prime} \subseteq A^{\prime}$ with $A \subseteq A^{\prime \prime}$. Suppose that $\Pi^{\prime} \in \mathcal{P}\left(G, A^{\prime \prime}\right)$ where either

(i) $\nu_{A^{\prime \prime}}\left(\mathcal{P}^{\prime}\right)>\nu_{A}(\mathcal{P})$ or

(ii) $\nu_{A^{\prime \prime}}\left(\mathcal{P}^{\prime}\right)=\nu_{A}(\mathcal{P})$ and there exists $(v, \beta) \in B_{A^{\prime \prime}}\left(\Pi^{\prime}\right)-\mathcal{R}(\mathcal{P}, A)$ where $v \notin D_{2}(\mathcal{P}, A)$.

Then, in $O\left(|V(G)|^{4}\right)$ time, we can find $\Pi \in \mathcal{P}(G, A)$ such that $\mathcal{P} \cup\{\Pi\}$ is richer than $\mathcal{P}$.

Proof. The proof is inductive on $\left|A^{\prime \prime}-A\right|$. If $A^{\prime \prime}=A$, then $\Pi:=\Pi^{\prime}$ satisfies the lemma. Thus we may assume that there exists $a \in A^{\prime \prime}-A$. Let $A_{1}=A^{\prime \prime}-\{a\}$. By Lemma 5.1, we can construct $\mathcal{P}_{1} \subset \mathcal{P}\left(G, A_{1}\right)$ such that either $\nu_{A_{1}}\left(\mathcal{P}_{1}\right)>$ $\nu_{A}(\mathcal{P})$ or $\nu_{A_{1}}\left(\mathcal{P}_{1}\right)=\nu_{A}(\mathcal{P})$ and $\mathcal{R}(\mathcal{P}, A) \subseteq \mathcal{R}\left(\mathcal{P}_{1}, A_{1}\right)$. Inductively, we may assume that $\nu_{A_{1}}\left(\mathcal{P}_{1}\right)=\nu_{A}(\mathcal{P}), D_{1}\left(\mathcal{P}_{1}, A_{1}\right)=D_{1}(\mathcal{P}, A)$, and $D_{2}\left(\mathcal{P}_{1}, A_{1}\right)=$ $D_{2}(\mathcal{P}, A)$. Now, by Lemma 4 .4, we can construct $\Pi^{\prime \prime} \in \mathcal{P}\left(G, A_{1}\right)$ such that $\mathcal{P}_{1} \cup\left\{\Pi^{\prime \prime}\right\}$ is richer than $\mathcal{P}_{1}$ with respect to $A_{1}$.

The next lemma is proved similarly; we leave the details to the reader.

Lemma 5.3. Let $X^{\prime} \subseteq X$. Suppose that $\Pi^{\prime} \in \mathcal{P}\left(G-X^{\prime}, A^{\prime}-X^{\prime}\right)$ where either (i) $\nu_{A^{\prime}-X^{\prime}}\left(\mathcal{P}^{\prime}\right)>\nu_{A}(\mathcal{P})$ or

(ii) $\nu_{A^{\prime}-X^{\prime}}\left(\mathcal{P}^{\prime}\right)=\nu_{A}(\mathcal{P})$ and there exists $(v, \beta) \in B_{A^{\prime}-X^{\prime}}\left(\Pi^{\prime}\right)-\mathcal{R}(\mathcal{P}, A)$ where $v \notin D_{2}(\mathcal{P}, A)$.

Then, in $O\left(|V(G)|^{4}\right)$ time, we can find $\Pi \in \mathcal{P}(G, A)$ such that $\mathcal{P} \cup\{\Pi\}$ is richer than $\mathcal{P}$.

Let $G_{1}=G-X$ and let $A_{1}=A^{\prime}-X$. Now, by Lemma 5.1, we can construct $\mathcal{P}_{1} \subset \mathcal{P}\left(G_{1}, A_{1}\right)$ such that either $\nu_{A_{1}}\left(\mathcal{P}_{1}\right)>\nu_{A}(\mathcal{P})$ or $\nu_{A_{1}}\left(\mathcal{P}_{1}\right)=\nu_{A}(\mathcal{P})$ and $\mathcal{R}(\mathcal{P}, A) \subseteq \mathcal{R}\left(\mathcal{P}_{1}, A_{1}\right)$. By Lemma 5.3, we may assume that $\nu_{A_{1}}\left(\mathcal{P}_{1}\right)=\nu_{A}(\mathcal{P})$, $D_{1}\left(\mathcal{P}_{1}, A_{1}\right)=D_{1}(\mathcal{P}, A)$, and $D_{2}\left(\mathcal{P}_{1}, A_{1}\right)=D_{2}(\mathcal{P}, A)$. Now let $G_{2}=G_{1}-$ $E_{0}\left(G_{1}, A_{1}\right)$. Note that no $A_{1}$-collection in $G_{1}$ uses an edge in $E_{0}\left(G_{1}, A_{1}\right)$, so $P_{1} \subseteq \mathcal{P}\left(G_{2}, A_{1}\right)$. Note that, if we can find $\Pi^{\prime} \in \mathcal{P}\left(G_{2}, A_{1}\right)$ such that $\operatorname{val}_{A_{1}}\left(\Pi^{\prime}\right)>\nu_{A_{1}}\left(\mathcal{P}_{1}\right)$, then, by Lemma 5.3 , we can construct $\Pi \in \mathcal{P}(G, A)$ such that $\mathcal{P} \cup\{\Pi\}$ is richer than $\mathcal{P}$. 
Let $H$ be a component of $G_{2}$, let $A_{H}=A_{1} \cap V(H)$. For each $\Pi \in \mathcal{P}\left(G_{2}, A_{1}\right)$, we let $\Pi \mid H$ denote the restriction of $\Pi$ to $H$ and let $\Pi-H$ denote the restriction of $\Pi$ to $G_{2}-H$. Let $\Pi_{1}, \Pi_{2} \in \mathcal{P}_{1}^{*}$. Suppose that $\operatorname{val}_{A_{H}}\left(\Pi_{1} \mid H\right)>$ $\operatorname{val}_{A_{H}}\left(\Pi_{2} \mid H\right)$. Now let $\Pi^{\prime}=\left(\Pi_{2}-H\right) \cup\left(\Pi_{1} \mid H\right)$. Note that $\Pi^{\prime} \in \mathcal{P}\left(G_{2}, A_{1}\right)$ and that $\operatorname{val}_{A_{1}}\left(\Pi^{\prime}\right)>\nu_{A_{1}}\left(\mathcal{P}_{1}\right)$, as required. Therefore we may assume that, for all $\Pi_{1}, \Pi_{2} \in \mathcal{P}_{1}^{*}$, we have $\operatorname{val}_{A_{H}}\left(\Pi_{1} \mid H\right)>\operatorname{val}_{A_{H}}\left(\Pi_{2} \mid H\right)$. Let $\mathcal{P}_{H}=\{\Pi \mid H$ : $\left.\Pi \in \mathcal{P}_{1}^{*}\right\}$.

Lemma 5.4. For each component $H$ of $G_{2}$, either $\operatorname{def}\left(H, A_{H}\right)=0$ or $\left(H, A_{H}\right)$ is $\mathcal{P}_{H}$-critical.

Proof. Note that, if $u v=e \in E(G)$ with $u \in V(G)-D(\mathcal{P}, A)$ and $v \in$ $D(\mathcal{P}, A)$, then either $u \in X$ or $e \in E_{0}\left(G, A^{\prime}\right)$. Moreover, $D\left(\mathcal{P}_{1}, A_{1}\right)=D(\mathcal{P}, A)$. Thus, if $H$ is a component of $G_{2}$, then either $V(H) \subseteq D\left(\mathcal{P}_{1}, A_{1}\right)$ or $V(H) \cap$ $D\left(\mathcal{P}_{1}, A_{1}\right)=\emptyset$. If $V(H) \cap D\left(\mathcal{P}_{1}, A_{1}\right)=\emptyset$, then $\operatorname{def}\left(\mathcal{P}_{H}, A_{H}\right)=0$. Thus we may assume that $V(H) \subseteq D\left(\mathcal{P}_{1}, A_{1}\right)$. Note that, since $H$ is a component of $G_{2}$, $D_{1}\left(\mathcal{P}_{H}, A_{H}\right)=D_{1}\left(\mathcal{P}_{1}, A_{1}\right) \cap V(H)$ and $D_{2}\left(\mathcal{P}_{H}, A_{H}\right)=D_{2}\left(\mathcal{P}_{1}, A_{1}\right) \cap V(H)$. By the definition of $A^{\prime}$, a vertex $v \in D\left(\mathcal{P}_{1}, A_{1}\right)$ is in $D_{1}\left(\mathcal{P}_{1}, A_{1}\right)$ if and only if $v \in A_{1}$. Hence $H$ is $\mathcal{P}_{H^{-}}$-critical, as required.

Suppose that $\left(H, A_{H}\right)$ is $\mathcal{P}_{H}$-critical and that $\operatorname{def}\left(\mathcal{P}_{H}, A_{H}\right)>1$. Then, by Lemma 4.5, we can construct $\Pi_{1} \in \mathcal{P}\left(H, A_{H}\right)$ such that $\operatorname{val}_{A_{H}}\left(\Pi_{1}\right)>$ $\nu\left(\mathcal{P}_{H}, A_{H}\right)$. Now let $\Pi_{2} \in \mathcal{P}_{1}^{*}$ and let $\Pi^{\prime}=\Pi_{1} \cup\left(\Pi_{2}-H\right)$. Note that $\Pi^{\prime} \in$ $\mathcal{P}\left(G_{2}, A_{1}\right)$ and that $\operatorname{val}_{A_{1}}\left(\Pi^{\prime}\right)>\nu_{A_{1}}\left(\mathcal{P}_{1}\right)$, as required. Therefore we may assume that: For each component $H$ of $G_{2}$, we have $\operatorname{def}\left(\mathcal{P}_{H}, A_{H}\right) \leq 1$. Thus $\operatorname{def}\left(G_{1}, A_{1}\right)=\operatorname{odd}\left(G_{1}, A_{1}\right)$. So, we have:

$$
\begin{aligned}
\operatorname{def}(\mathcal{P}, A) & =\operatorname{def}\left(G-X, A^{\prime}-X\right)-\left|A^{\prime}-A\right|-|X| \\
& =\operatorname{odd}\left(G-X, A^{\prime}-X\right)-\left|A^{\prime}-A\right|-|X|,
\end{aligned}
$$

as required. This completes the description and proof of the algorithm.

Let $n=|V(G)|$. The algorithm, as stated, requires $O\left(n^{6}\right)$ time. The complexity in Lemma 3.2 can be improved from $O\left(n^{3}\right)$ to $O\left(n^{2}\right)$, by combining the proofs of Lemma 3.2 and 3.1. This reduces the overall complexity of our algorithm from $O\left(n^{6}\right)$ to $O\left(n^{5}\right)$.

\section{Acknowledgements}

We thank Bert Gerards for his contributions towards the formulation and proof of Theorem 1.3. We also thank the anonymous referee for their careful reading of the manuscript. 


\section{References}

[1] M. Chudnovsky, J. Geelen, B. Gerards, L. Goddyn, M. Lohman and P. SeyMOUR: Packing non-zero $A$-paths in group-labelled graphs, Combinatorica 26(5) (2006), 521-532.

[2] L. LovÁsz: Matroid matching and some applications, J. Combin. Theory Ser. B 28 (1980), 208-236.

[3] L. Lovász and M. D. Plummer: Matching Theory, North-Holland, 1986.

[4] W. Mader: Über die Maximalzahl kreuzungsfreier $H$-Wege, Archiv der Mathematik (Basel) 31 (1978), 382-402.

[5] A. Sebö and L. Szegö: The path-packing structure of graphs, in: Integer Programming and Combinatorial Optimization (Proceedings 10th IPCO Conference, New York, 2004; D. Bienstock, G. Nemhauser, eds.) [Lecture Notes in Computer Science 3064], Springer, Berlin, 2004, pp. 256-270.

Maria Chudnovsky

Department of Industrial Engineering and Operations Research

Columbia University

New York, NY 10027

$U S A$

\section{Jim Geelen}

Department of Combinatorics and Optimization

University of Waterloo

Waterloo, N2L $3 G 1$

Canada

\section{William H. Cunningham}

Department of Combinatorics and Optimization University of Waterloo

Waterloo, N2L $3 G 1$

Canada 